\title{
Enhancement of Output Power for GaN-Based LEDs by Treatments of Ar Plasma on p-GaN Surface
}

\author{
X. F. Zeng, ${ }^{1}$ S. C. Shei, ${ }^{2}$ H. M. Lo, ${ }^{3}$ and S. J. Chang ${ }^{1}$ \\ ${ }^{1}$ Institute of Microelectronics and Department of Electrical Engineering, Advanced Optoelectronic Technology Center, \\ Research Center for Energy Technology and Strategy, National Cheng Kung University, 1 University Road, Tainan 701, Taiwan \\ ${ }^{2}$ Department of Electrical Engineering, National University of Tainan, No. 33, Section 2, Shu-Lin Street, Tainan 70005, Taiwan \\ ${ }^{3}$ Department of R \& D, Aceplux Optotech Incooperation, 1 Hsing-Horng Road, Tainan 708, Taiwan
}

Correspondence should be addressed to S. C. Shei; scshei@mail.nutn.edu.tw

Received 31 August 2013; Revised 18 October 2013; Accepted 18 October 2013

Academic Editor: Liang-Wen Ji

Copyright (C) 2013 X. F. Zeng et al. This is an open access article distributed under the Creative Commons Attribution License, which permits unrestricted use, distribution, and reproduction in any medium, provided the original work is properly cited.

\begin{abstract}
We successfully demonstrated that the Arplasmatreatment p-GaN surface increased the contact resistance of ITO/P-GaN serving as injection current deflection layer under the electrode pad. It was found that the $V_{f}$ values of the two LEDs at $20 \mathrm{~mA}$ were approximately $3.3 \mathrm{~V}$. Under a $20 \mathrm{~mA}$ current injection, it was found that output powers of conventional LED and Ar-plasmatreatment LED on p-GaN surfaces were 9.8 and $11.08 \mathrm{~mW}$, respectively. We can increase the output power of GaN LEDs in $13 \%$ due to current blocking on the surface of $\mathrm{p}-\mathrm{GaN}$ under the electrode pad by inserting the treatment with Ar plasma. It was also found that, after the reliability test for 72 hours the half lifetimes of conventional LEDs and LEDs with Ar-plasma treatment on p-GaN surface were about $49 \%$ and $55 \%$, corresponding to the initial intensity, respectively.
\end{abstract}

\section{Introduction}

Nitride-based materials have recently emerged as important semiconductor materials, leading to the realization of high performance light emitters from ultraviolet (UV) to blue and green spectral regions [1,2]. For example, GaN-based blue and green light-emitting diodes (LEDs) have already been extensively used in full-color displays and as highly efficient light sources for traffic-light lamps. Highefficiency nitride-based LEDs are also potentially useful for solid state lighting. However, the external quantum efficiency (EQE) of LEDs, which is defined as the product of internal quantum efficiency and light-extraction efficiency, is still required to be further improved. To improve the EQE of LEDs, the internal quantum efficiency and light-extraction efficiency must be increased. To approach solid state lighting, however, one needs to further improve the output efficiency of these LEDs. There are several parameters that affect the output efficiency of the LEDs, such as light extraction efficiency, internal quantum efficiency, and current distribution of LEDs. It has been demonstrated that several methods can be used to improve output efficiency of GaN-based LEDs by enhancing the lightextraction efficiency, such as textured surfaces [3-5], a highly transparent p-contact layer [6], a proper substrate design [7], and flip-chip packaging [8]. Moreover, the crystal quality, device band engineering structure design, and doping profile would affect the internal quantum efficiency of LEDs. Besides these factors, current distributed throughout the LEDs also plays one of the key roles in the efficiency of the LEDs as well. In other words, current distributed throughout the LEDs determines the real light-emitting area of the devices, and light output power is proportional to the light-emitting area. Recently, there are several approaches for improving the current spreading and the light-extraction efficiency of GaNbased LEDs by current blocking layer (CBL) $[9,10]$. Current distribution had been wildly studied in the GaAs-based LEDs [11-13] to increase output power. Current blocking is one of the ways to change current distribution in the LEDs. The current injected by the top contact enters the active region predominantly under the top contact. The extraction 
of the light generated in the active region is thus strongly hindered by the opaque metal contact. The current block layer would block the entering current from top contact to the active region. The light-extraction efficiency of LEDs is improved because the current is deflected away from the top contact. In this study, we would apply the different methods of current-blocking layer formation on the InGaN/GaN multiple quantum wells (MQWs) LEDs. We would also compare the electrical, optical and reliability characteristics of those different current blocking layers.

\section{Experimental}

Samples used in this study were all grown on 2-inch (0001) sapphire substrate by vertical metal organic chemical vapor deposition (MOCVD). The detailed layers and growth procedures have been described in a previous publication [13]. The thicknesses of the p-AlGaN and p-GaN layers were $50 \mathrm{~nm}$ and $150 \mathrm{~nm}$, respectively. After growing the samples, postannealing was needed to activate the top $\mathrm{p}-\mathrm{GaN}$ layer of InGaN/GaN MQWs LED at $700^{\circ} \mathrm{C}$ and $20 \mathrm{~min}$. The partial $\mathrm{p}-\mathrm{GaN}$ and active layers were removed to expose the $\mathrm{n}-\mathrm{GaN}$ layer for the n-electrode ohmic contact by using the Inductive Coupled Plasma (ICP) etcher. The current blocking layer was formed right before the deposition of the indiumtinoxide (ITO) transparent contact layer (TCL). An Ar-plasma treatment on surface of $\mathrm{p}-\mathrm{GaN}$ underneath the p-electrode pad was introduced to form the current-blocking layer in this study with the following conditions: Ar: $20 \mathrm{sccm}$, working pressure: 3 mtorr, and ICP power: $120 \mathrm{~W}$. Then, the ITO TCL was deposited on the $\mathrm{p}-\mathrm{GaN}$ surface to cover the current blocking layer. The $\mathrm{Cr} / \mathrm{Au}(50 / 200 \mathrm{~nm})$ bilayer metal contact was deposited on the ITO TCL and exposed $\mathrm{n}^{+}-\mathrm{GaN}$ layers to form the p-type electrode (anode) and the n-type electrode (cathode), respectively, at the same time. The chip size of the LEDs in our study was $8 \times 10 \mathrm{mil}^{2}$. The conventional LED is LED I and the ICP plasma treatment LED is LED II in the paper. The current-voltage $(I-V)$ characteristics of the current blocking layer and experimental LEDs were measured by using the HP-4156C semiconductor parameter analyzer, and the output powers of the LEDs were measured by using calibrated integrating sphere.

\section{Results and Discussion}

Figure 1 indicated the $I-V$ characteristics of the ITO/p-GaN contact with and without Ar plasma treatment on p-GaN surface. It was found that the resistance of $\mathrm{ITO} / \mathrm{p}-\mathrm{GaN}$ contact increased after the Ar plasma treatment of the $\mathrm{p}-\mathrm{GaN}$ surface. The ITO/p-GaN contact with Ar plasma treatment on p-GaN surface was shown as a Schottky-like contact and the ITO/p-GaN contact without the plasma treatment was exhibited as an ohmic-like contact. $I-V$ characteristics of the two curves in Figure 1 do not show Schottky characteristic or perfect ohmic characteristic. The resistances of the contact without and after Ar plasma are $3.1 \times 10^{-3} \Omega \mathrm{cm}^{2}$ and $5.0 \times$ $10^{-1} \Omega \mathrm{cm}^{2}$. It was found that the resistance of the contact after Ar plasma is larger than that of the conventional contact.

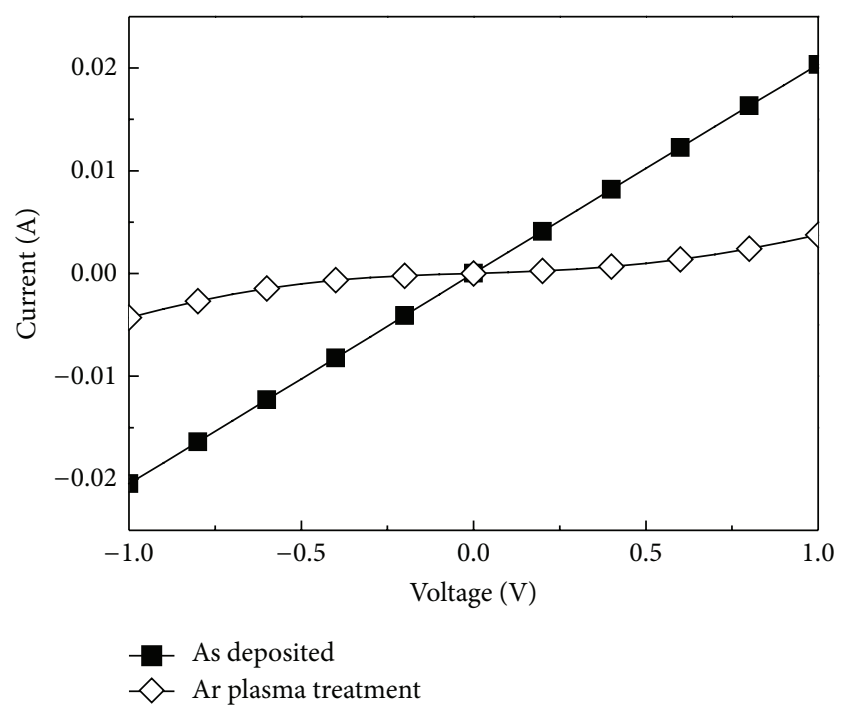

FIGURE 1: The $I-V$ curves of the ITO/p-GaN contact with Ar plasma treatment on $\mathrm{p}-\mathrm{GaN}$ surface and without the treatment.

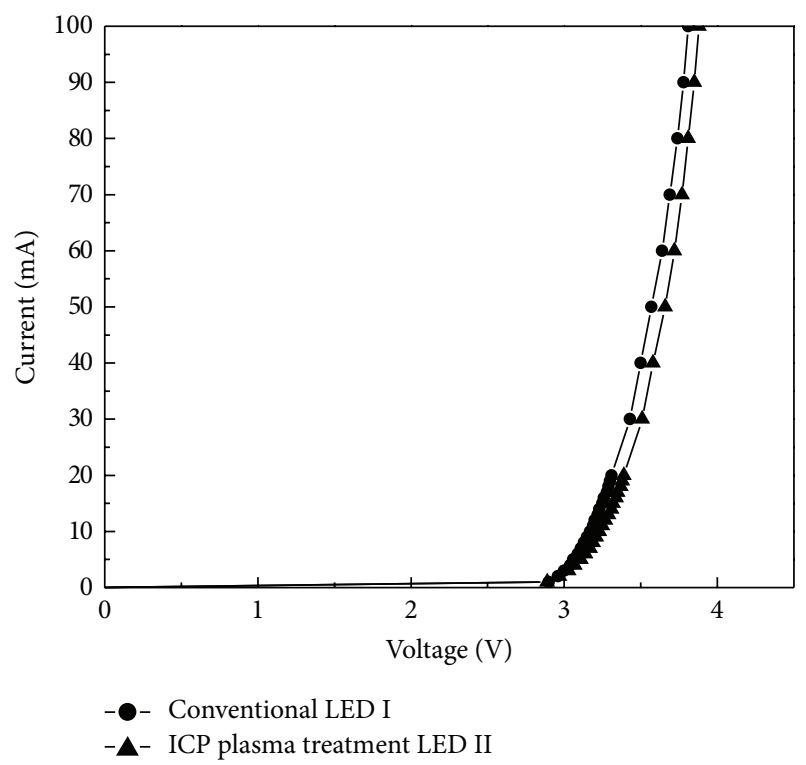

FIgURE 2: The current-voltage curves of LEDs with and without (i.e. ICP plasma treatment LED II and conventional LED I, resp.) Ar plasma treatment under electrode p-pad.

The increase of the resistance of the ITO/p-GaN contact on Ar plasma treatment $\mathrm{p}-\mathrm{GaN}$ surface should be attributed to the Ar plasma damage resulting in a decrease the carrier concentration on the $\mathrm{p}-\mathrm{GaN}$ surface. It should be noted that high contact resistance of the Ar plasma-damaged p-GaN surface could be served as the current deflection resistance right under the electrode pads.

Figure 2 indicated the current-voltage $(I-V)$ curve of LEDs with and without (i.e., ICP plasma treatment LED: LED II and conventional LED: LED I, resp.) Ar plasma treatment under the electrode pad. As shown in Figure 2, it was found that the forward voltage, $\left(V_{f}\right)$ of conventional LED I and 


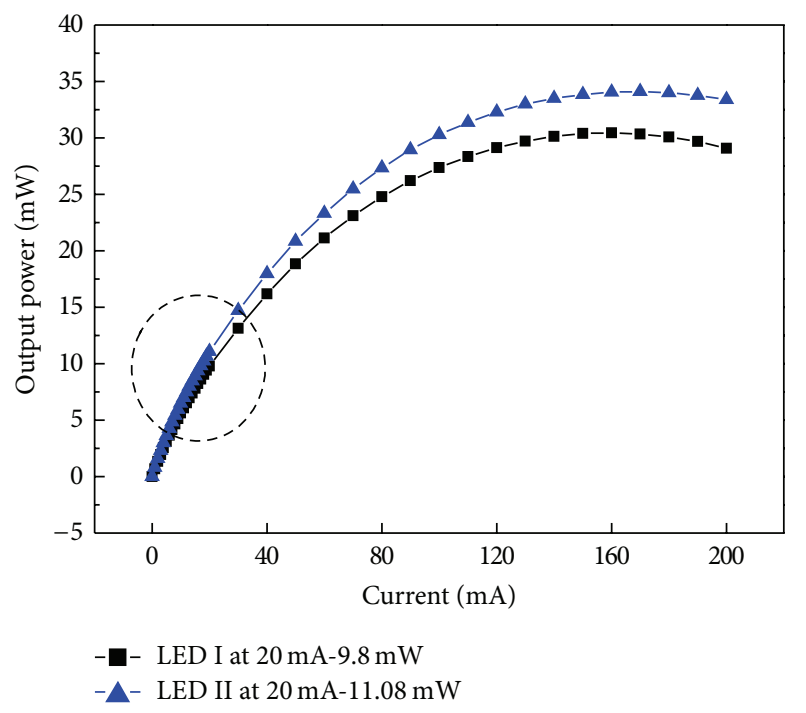

FIGURE 3: The light output powers of GaN-based LED for conventional and with ICP plasma treatment, respectively.

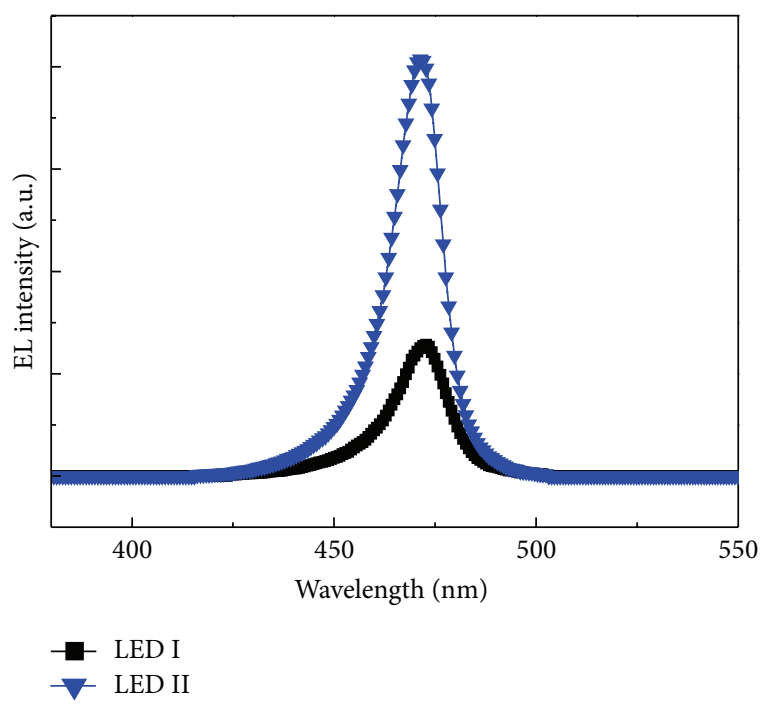

FIGURE 4: The EL of GaN-based LED for conventional and with ICPplasma treatment, respectively.

Ar-plasma-treatment LED II at $20 \mathrm{~mA}$ were approximately 3.31 and $3.39 \mathrm{~V}$, responsively. The lightly increase in $20 \mathrm{~mA}$ $V_{f}$ results from the increase in current density for Arplasma-treatment LED-II. The $20 \mathrm{~mA}-V_{f}$ results indicated that the electrical characteristics of LEDs were not obviously degraded by the Ar plasma treatment under the electrode pad processes.

Figure 3 indicated the intensity-voltage $(L-I)$ curves of LEDs with and without Ar plasma treatment under the electrode pad. Furthermore, it was found that output powers of these two LEDs were increased as we increased the injection current. Under the same injection current, it was found that the output power observed from ICP-plasma treatment LED II was always larger than the conventional
LED I. Figure 3 showed that the output powers were 9.8 and $11.08 \mathrm{~mW}$ for conventional LED I and ICP-plasma treatment LED II under $20 \mathrm{~mA}$ current injections, respectively. In other words, we can increase the LED output power by $13 \%$ by inserting the Ar plasma-damaged p-GaN surface under the electrode pad. The enhancement of output power observed from ICP-plasma treatment LED II should be attributed to the improved current spreading of LEDs by high contact resistance of the ITO/p-GaN under electrode pad. To further investigate the effects of current distribution, we measured near field optical images of the LEDs.

Figure 4 shows the EL spectra of a conventional LED I and ICP-plasma-treatment LED II at an injection current of $20 \mathrm{~mA}$. No significant differences in the EL peak position at $446 \mathrm{~nm}$ were detected throughout the three types of LEDs. However, the EL intensity of LED II was higher than that of LED I.

Figure 5 shows the 3D images measured from the LED I and LED II at $20 \mathrm{~mA}$ DC injection current into the devices. It can be seen clearly that the output light was emitted uniformly across the chips since current spread uniformly in LED II. In contrast, it was found that light output distribution is nonuniform for the conventional LED I. The better current spreading of LED II should be attributed to the high ITO/pGaN contact resistance under the electrode pad deflecting the injection current away from pad and then increasing the emission area of devices. In other words, the LED I without high resistive Ar plasma-damaged p-GaN surface under electrode pad would lead the most of injection current to go into devices under the electrode pad. However, the light emitted under the pad would be absorbed by the opaque metal pad and then reduce the output power of LED. At an injection current of $20 \mathrm{~mA}$, the light output power of LEDs with Ar plasma treatment was 13\% larger than that of conventional LEDs. At an injection current of $100 \mathrm{~mA}$, the temperature of the p-pad metal on LEDs with Ar plasma treatment is $13^{\circ} \mathrm{C}$ lower than that of the LEDs with a Arplasma CBL. However, the output power of LEDs with Ar plasma treatment is the lower due to the surface damage of $\mathrm{p}$-GaN under the p-pad electrode. Besides, the non-uniform current distribution near the electrode pad might enhance the current crowding while driving at high current. Therefore, it was found that the injection current to reache the highest output power of conventional LED I is less than that of ICPplasma treatment LED II in Figure 2.

Figure 6 shows the reliability test of the LED I and II injection with $50 \mathrm{~mA}$ at $80^{\circ} \mathrm{C}$. It was found that the decayed intensities of LED I and LED II were about $49 \%$ and 55\% of the initial intensity, respectively, after aging 72 hours. It should also be attributed to the better current spreading of the LED II with high ITO/p-GaN contact resistance under the electrode pad. The better current spreading could reduce the current crowding under the pad at high current injection. As a result, the intensity degradation of the LED II during the high temperature and high injection current aging is less than that of LED I. The decrease in optical power was closely correlated to an increase in operating voltage due to an increase in the parasitic series resistance during the accelerated current aging test. 


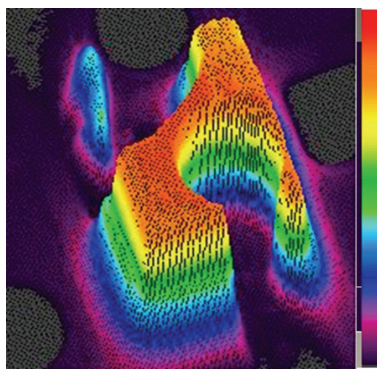

(a) With Ar plasma treatment

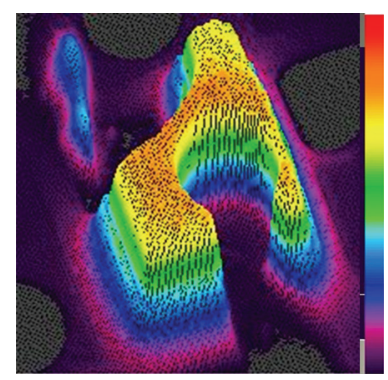

(b) Without Ar plasma treatment

FIGURE 5: (a) and (b) show the 3D images measured from the LEDs with and without Ar plasma-damaged p-GaN surface under electrode p-pad, respectively.

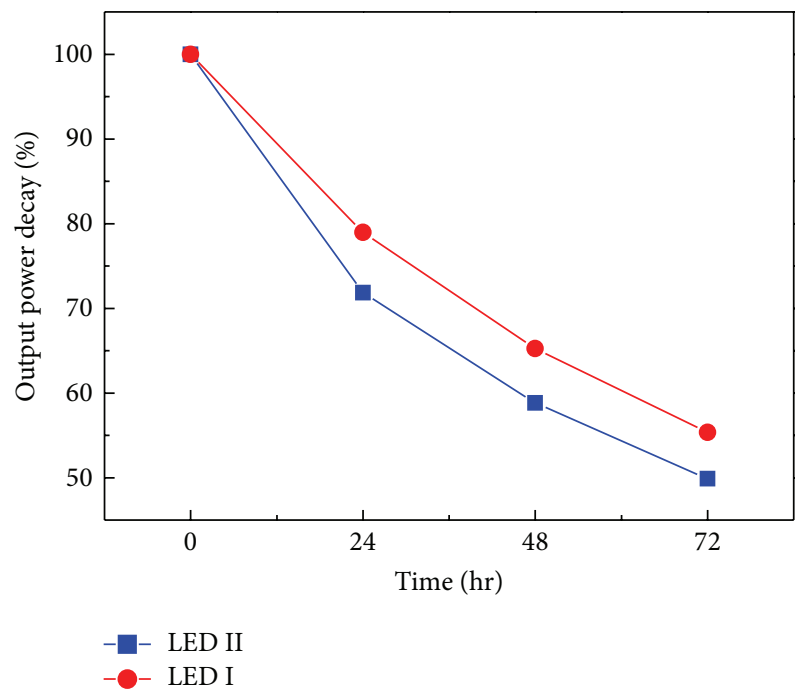

FIGURE 6: Reliability tests of the LED I and II injection with $50 \mathrm{~mA}$ at $80^{\circ} \mathrm{C}$.

\section{Conclusion}

In summary, we successfully demonstrated that the $\mathrm{Ar}$ plasma-damaged $\mathrm{p}-\mathrm{GaN}$ surface increased the resistance of ITO/P-GaN contact serving as injection current deflection layer under the electrode pad. It was found that the $V_{f}$ of both LEDs at $20 \mathrm{~mA}$ were approximately $3.3 \mathrm{~V}$. It was found that output powers were 9.8 and $11.08 \mathrm{~mW}$ for LED I and LED II at $20 \mathrm{~mA}$, respectively. In other words, we can increase the LED output power by $13 \%$ by inserting the Ar plasmadamaged $\mathrm{p}-\mathrm{GaN}$ surface under the electrode pad. It was also found that the decayed intensities of LED I and LED II were about $49 \%$ and $55 \%$ of the initial intensity, respectively, after aging 72 hours. It was believed that high resistance ITO/P$\mathrm{GaN}$ contact with Ar plasma-damaged $\mathrm{p}-\mathrm{GaN}$ surface under pad would improve the injection current spreading to the whole device. And better current spreading could lead to improving the photoelectrical characteristics of LEDs.

\section{Acknowledgments}

This work was granted in part by the Center for Frontier Materials and Micro/Nano Science and Technology and in part by the Advanced Optoelectronic Technology Center, National Cheng Kung University, under projects from the Ministry of Education, Taiwan. This work was also supported in part by the Ministry of Economic Affairs (MOEA) NSC 98-2622-E-024-001-CC3 and NSC 99-2221-E-024-009. The authors also would like to thank the Bureau of Energy, Ministry of Economic Affairs of Taiwan, for financially supporting this research under Contract no. 98-D0204-6, and the LED Lighting and Research Center, NCKU, for the assistance in related measurements.

\section{References}

[1] T. Mukai, M. Yamada, and S. Nakamura, "Characteristics of InGaN-based UV/blue/green/amber/red light-emitting diodes," Japanese Journal of Applied Physics, vol. 38, no. 7, pp. 3976-3981, 1999.

[2] T. Mukai, M. Yamada, and S. Nakamura, "Current and temperature dependences of electroluminescence of InGaNbased UV/blue/green light-emitting diodes," Japanese Journal of Applied Physics, vol. 37, no. 11, pp. L1358-L1361, 1998.

[3] T. Fujii, Y. Gao, R. Sharma, E. L. Hu, S. P. DenBaars, and S. Nakamura, "Increase in the extraction efficiency of GaN-based light-emitting diodes via surface roughening," Applied Physics Letters, vol. 84, no. 6, pp. 855-857, 2004.

[4] S.-I. Na, G.-Y. Ha, D.-S. Han et al., "Selective wet etching of p-GaN for efficient GaN-based light-emitting diodes," IEEE Photonics Technology Letters, vol. 18, no. 14, pp. 1512-1514, 2006.

[5] C. H. Kuo, C. C. Lin, S. J. Chang et al., "Nitride-based light-emitting diodes with p-AIInGaN surface layers," IEEE Transactions on Electron Devices, vol. 52, no. 10, pp. 2346-2349, 2005.

[6] S. J. Chang, C. S. Chang, Y. K. Su et al., "Nitride-based LEDs with an SPS tunneling contact layer and an ITO transparent contact," IEEE Photonics Technology Letters, vol. 16, no. 4, pp. 1002-1004, 2004.

[7] C. H. Kuo, S. J. Chang, Y. K. Su et al., "Nitride-based nearultraviolet LEDs with an ITO transparent contact," Materials Science and Engineering B, vol. 106, no. 1, pp. 69-72, 2004. 
[8] C.-J. Tun, J.-K. Sheu, B.-J. Pong et al., "Enhanced light output of GaN-based power LEDs with transparent Al-doped $\mathrm{ZnO}$ current spreading layer," IEEE Photonics Technology Letters, vol. 18, no. 1, pp. 274-276, 2006.

[9] C. Huh, J.-M. Lee, D.-J. Kim, and S.-J. Park, "Improvement in light-output efficiency of InGaN/GaN multiple-quantum well light-emitting diodes by current blocking layer," Journal of Applied Physics, vol. 92, no. 5, pp. 2248-2250, 2002.

[10] M.-A. Tsai, P. Yu, J. R. Chen et al., "Improving light output power of the GaN-Based vertical-injection light-emitting diodes by $\mathrm{Mg}^{+}$implanted current blocking layer," IEEE Photonics Technology Letters, vol. 21, no. 11, pp. 688-690, 2009.

[11] J. Nishizawa, M. Koike, and C. C. Jin, "Efficiency of GaAlAs heterostructure red light-emitting diodes," Journal of Applied Physics, vol. 54, no. 5, pp. 2807-2812, 1983.

[12] C. P. Kuo, R. M. Fletcher, T. D. Osentowski, M. C. Lardizabal, M. G. Craford, and V. M. Robbins, "High performance AlGaInP visible light-emitting diodes," Applied Physics Letters, vol. 57, no. 27, pp. 2937-2939, 1990.

[13] H. Sugawara, M. Ishikawa, and G. Hatakoshi, "High-efficiency InGaAlP/GaAs visible light-emitting diodes," Applied Physics Letters, vol. 58, no. 10, pp. 1010-1012, 1991. 

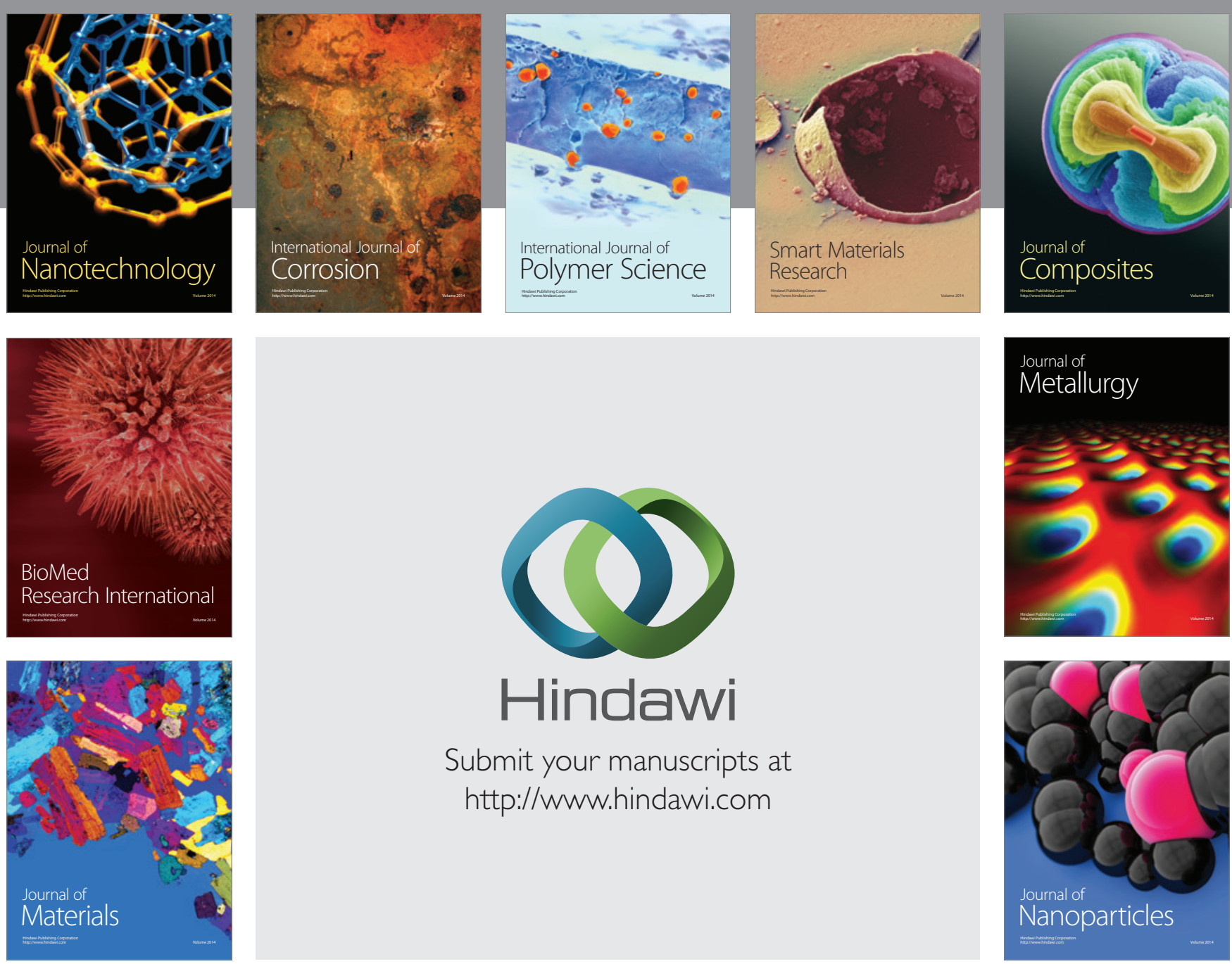

Submit your manuscripts at http://www.hindawi.com
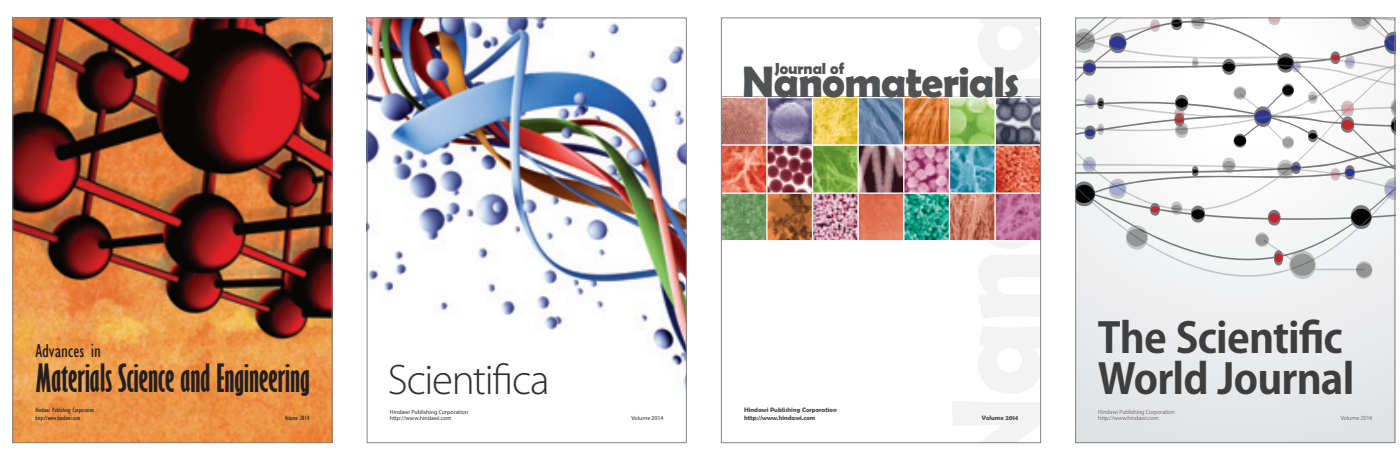

\section{The Scientific World Journal}
